\title{
Cyst Nematode Parasitism of Arabidopsis thaliana Is Inhibited by Salicylic Acid (SA) and Elicits Uncoupled SA-Independent Pathogenesis-Related Gene Expression in Roots
}

\author{
Martin John Evers Wubben, ${ }^{1}$ Jing Jin, ${ }^{2}$ and Thomas Josef Baum ${ }^{2}$ \\ ${ }^{1}$ United States Department of Agriculture-Agricultural Research Service, Crop Science Research Laboratory, \\ Mississippi State, MS 39762, U.S.A.; ${ }^{2}$ Department of Plant Pathology, lowa State University, Ames, IA 50011, U.S.A.
}

Submitted 22 June 2007. Accepted 13 November 2007.

Compatible plant-nematode interactions involve the formation of an elaborate feeding site within the host root that requires the evasion of plant defense mechanisms by the parasite. Little is known regarding plant defense signaling pathways that limit nematode parasitism during a compatible interaction. Therefore, we utilized Arabidopsis thaliana mutants perturbed in salicylic acid (SA) biosynthesis or signal transduction to investigate the role of $\mathrm{SA}$ in inhibiting parasitism by the beet cyst nematode Heterodera schachtii. We determined that SA-deficient mutants (sid2-1, pad4-1, and NahG) exhibited increased susceptibility to $H$. schachtii. In contrast, SA-treated wild-type plants showed decreased $H$. schachtii susceptibility. The npr1-2 and npr1-3 mutants, which are impaired in SA signaling, also showed increased susceptibility to $H$. schachtii, whereas the nprlsuppressor mutation snil showed decreased susceptibility. Constitutive pathogenesis-related (PR) gene-expressing mutants (cpr1 and cpr6) did not show altered susceptibility to $H$. schachtii; however, constitutive PR gene expression was restricted to cprl shoots with wild-type levels of PR-1 transcript present in cpr1 roots. Furthermore, we determined that $H$. schachtii infection elicits SA-independent PR-2 and PR-5 induction in wild-type roots, while PR-1 transcript and total SA levels remained unaltered. This was in contrast to shoots of infected plants where PR-1 transcript abundance and total SA levels were elevated. We conclude that SA acts via NPR1 to inhibit nematode parasitism which, in turn, is negatively regulated by SNI1. Our results show an inverse correlation between root basal PR-1 expression and plant susceptibility to $H$. schachtii and suggest that successful cyst nematode parasitism may involve a local suppression of SA signaling in roots.

Sedentary endoparasitic nematodes are obligate biotrophs that induce the formation of complex feeding sites within the roots of their plant host. This group of plant pathogens includes the

Corresponding authors: M. J. E. Wubben; Telephone: +1.662.320.7585; Fax: +1.662.320.7528; E-mail: Martin.Wubben@ars.usda.gov; and T. J. Baum; Telephone: +1.515.294.5420; Fax: +1.515.294.9420; E-mail: tbaum@iastate.edu

This article is in the public domain and not copyrightable. It may be freely reprinted with customary crediting of the source. The American Phytopathological Society, 2008. cyst nematodes (Heterodera and Globodera spp.), which induce the formation of a group of fused cells called a syncytium in proximity to the root vasculature, and the root-knot nematodes (Meloidogyne spp.), which induce and feed from giant cells (Williamson and Gleason 2003). Taken together, cyst and rootknot nematodes account for $>\$ 100$ billion in annual yield losses worldwide on an enormous variety of crops (Sasser and Freckman 1987). Because a living plant cell is required to act as an initial feeding cell, these pathogens have evolved mechanisms to avoid or negate both preexisting and inducible plant defense mechanisms in susceptible host plants in order to minimize cell damage and promote feeding site development.

Due to the economic damage imposed by sedentary endoparasitic nematodes on agriculturally important plants, there is much interest in identifying and understanding the resistance mechanisms employed by crop hosts that are able to limit nematode reproduction. Gene-for-gene relationships between plant hosts and sedentary endoparasitic nematodes have been described and the corresponding plant resistance gene ( $R$ gene) identified in some cases (Williamson and Kumar 2006). Although much attention has been given toward understanding the signaling mechanisms that underlie incompatible plantnematode interactions, relatively little is known regarding defense mechanisms employed during compatible plant-nematode interactions. As an alternative to $R$-gene-mediated resistance, the identification of defense-related genes and their respective signaling pathways that act to limit nematode parasitism during a compatible interaction may lend themselves to bioengineering and provide an additional layer of defense that can be introduced into crop plants.

A number of studies have been conducted using cDNA- or oligo-based microarrays with the goal of identifying plant genes that are differentially regulated by the nematode during a compatible interaction. A gene expression survey of giantcell formation in Arabidopsis thaliana roots following infection by Meloidogyne incognita suggested that successful rootknot nematode infection involves a local suppression of host defense signaling (Jammes et al. 2005). In contrast, a broad general activation of defense signaling was observed during soybean cyst nematode infection of soybean (Ithal et al. 2007). Analysis of whole A. thaliana roots infected with either Heterodera schachtii or $H$. glycines revealed a number of defenserelated genes that were differentially expressed in the root following infection compared with the noninoculated control (Puthoff et al. 2003). These experiments revealed a definite involvement of defense signaling pathways during compatible 
plant-nematode interactions; however, the details of which defense pathways are actually effective against sedentary endoparasitic nematodes remain unresolved.

Plant defense signaling has been well studied in the model plant A. thaliana, where large emphasis has been placed on dissecting the signaling pathways that govern salicylic acid (SA) accumulation and subsequent defense gene expression. SA regulates many aspects of the plant defense response, including the development of systemic acquired resistance (SAR), disease development during compatible interactions, and, in some instances, $R$-gene-mediated resistance (Delaney et al. 1994; Gaffney et al. 1993; Nawrath and Métraux 1999; Wildermuth et al. 2001). Local and systemic SA accumulation following pathogen infection induces the expression of multiple pathogenesis-related (PR) genes (PR-1, PR-2, and PR-5) whose inductions commonly are used as molecular markers for the activation of the SA signaling pathway and the onset of SAR in A. thaliana (Durrant and Dong 2004; Uknes et al. 1992). SA necessary for plant defense is believed to be synthesized through the action of SID2, an isochorismate synthase gene. Loss-of-function mutations within SID2 render plants unable to accumulate SA following pathogen infection (Wildermuth et al. 2001). The mutants sidl-1 (also known as eds5-1) and sid21 (also known as eds16) are more susceptible to bacterial and fungal pathogens compared with wild-type plants (Dewdney et al. 2000; Nawrath and Métraux 1999; Rogers and Ausubel 1997). Mutant pad4-1 shows compromised SA accumulation due to a loss-of-function mutation in a lipase-like gene (Jirage et al. 1999). Finally, compromised SA accumulation also can be achieved in wild-type plants by the transgenic expression of $N a h G$, the gene for a bacterial salicylate hydroxylase that degrades SA to catechol (Gaffney et al. 1993).

NPR1 is a key component of the SA signaling pathway, such that genetic screens designed to identify mutants insensitive to SA treatment recovered multiple $n p r l$ alleles (Cao et al. 1994; Delaney et al. 1995; Shah et al. 1997). nprl mutant plants do not show PR gene induction following treatment with SA or any of its functional analogues and, in many instances, show increased susceptibility to virulent pathogens (Cao et al. 1994; Delaney et al. 1995; Glazebrook et al. 1996; McDowell et al. 2005). The position of NPR1 within the SA signal transduction pathway is well established and genes have been identified that act downstream of NPR1 to regulate defense gene expression. For example, a screen designed to identify suppressors of the nprl mutation identified the snil mutant ( $\mathrm{Li}$ et al. 1999). In addition to mutants that are defective in SA responsiveness, mutants that constitutively express PR genes (cprl, cpr5, and cpr6) also have been identified. Such mutants generally show increased resistance to pathogens relative to wild-type plants (Bowling et al. 1994, 1997; Clarke et al. 1998). The sheer number and unique properties of these various mutants provides an opportunity to assess many facets of SA-mediated defense signaling as it relates to the Arabidopsis-nematode compatible interaction.

Studies have been conducted regarding the effect of exogenously applied SA on plant-parasitic nematode reproduction. Root drenching of white clover with SA prior to inoculation with the clover cyst nematode, $H$. trifolii, resulted in decreased nematode reproduction (Kempster et al. 2001). Also, application of SA as a foliar spray to okra and cowpea caused a decrease in root-knot nematode infection (Nandi et al. 2003). However, in this report, we present an in-depth characterization of the role of SA in mediating effective Arabidopsis defense mechanisms to the root pathogen $H$. schachtii, the sugar beet cyst nematode. We show that SA-mediated signaling governed by NPR1 and negatively regulated by SNI1 limits cyst nematode parasitism during a compatible interaction. We also demonstrate that cyst nematode infection elicits an unusual pattern of PR gene expression in Arabidopsis roots, such that PR-2 and PR-5 but not PR-1 are induced following nematode infection. In shoots, however, elevated PR-1 transcript levels were detected in infected plants relative to the control. This dichotomy in PR-1 expression between shoots and roots of infected plants also was present in our finding that total SA levels were unaltered in infected roots but were elevated in shoots of infected plants. We further determined that PR-2 and PR-5 induction by the nematode occurs in an SA-independent fashion. These results suggest that successful cyst nematode parasitism of A. thaliana may involve a suppression of the local SA signaling pathway in roots.

\section{RESULTS}

\section{The SA signaling pathway \\ inhibits cyst nematode parasitism.}

It has been well established that SA plays a critical role in plant defense signaling. To determine whether SA affects cyst nematode parasitism during a compatible interaction, we investigated the effects of SA deficiency and SA treatment on A. thaliana susceptibility to the sugar beet cyst nematode $H$. schachtii.

A number of mutants have been identified that are defective in pathogen-induced SA accumulation. For our experiments, we chose to evaluate the $H$. schachtii susceptibility of four such mutants: sid1-1, sid2-1, pad4-1, and the transgenic NahG line. The susceptibilities of sid1-1, sid2-1, pad4-1, NahG, and a wild-type Columbia-0 (WT) control were determined at 2 weeks after inoculation with infective $H$. schachtii secondstage juveniles (J2), at which time numbers of female nematodes were used as a direct measure of plant susceptibility. Root development and rate of root growth appeared similar between all the tested genotypes.

We observed a significant increase in $H$. schachtii susceptibility for sid2-1, pad4-1, and NahG compared with WT (Fig. 1A). In contrast, the sid1-1 mutant did not exhibit any appreciable difference in susceptibility compared with WT (Fig. 1A). It should be noted that all previous characterizations of sidl-1 were conducted with shoot tissues, whereas $H$. schachtii is a root pathogen. Furthermore, the precise role of SID1 in SA biosynthesis remains undetermined (Nawrath et al. 2002). Therefore, the observed lack of increased susceptibility in sidl-1 to $H$. schachtii may suggest that SID1 plays a minor role in SA accumulation as it relates to root tissues. In addition, although it has been demonstrated that the by-product of SA degradation via $N a h G$ (i.e., catechol) can itself compromise defense responses (Glazebrook et al. 2003; Heck et al. 2003), the observation that two additional mutants (sid2-1 and pad4-1) show increased susceptibility to $H$. schachtii supports the conclusion that the increased susceptibility of $N a h G$ is due to changes in SA or SAmediated signaling and not increased catechol concentrations.

Following these initial results, we further investigated the inhibitory role of SA on $A$. thaliana susceptibility to $H$. schachtii by treating WT plants with SA prior to inoculation with $H$. schachtii. WT plants were germinated and grown on control medium or medium containing $50 \mu \mathrm{M}$ SA and then inoculated with $H$. schachtii J2 10 to 12 days after planting. We observed that WT plants grown on SA-containing medium exhibited a marked decrease in susceptibility to $H$. schachtii compared with those grown on control medium (Fig. 1B). We also determined that SA by itself does not appear to have a detrimental effect on $H$. schachtii infectivity. Inoculation of WT plants with J2 nematodes that had been incubated overnight in a $50 \mu \mathrm{M}$ SA or control solution resulted in the development of similar mean \pm standard error numbers of $\mathrm{J} 4$ female nematodes ( $4.9 \pm 1.1$ and $5.0 \pm 0.6$, respectively). Elevated PR-1 gene transcript levels were detected in roots of SA-treated but 
not control plants by quantitative real-time reverse-transcriptase polymerase chain reaction (qRT-PCR) prior to nematode inoculation (data not shown). The finding that SA incubation does not harm $H$. schachtii $\mathrm{J} 2$ and that SA treatment of WT plants decreases the number of female nematodes that are able to develop supports the conclusion that SA-mediated signaling is a significant inhibitor of $A$. thaliana susceptibility to $H$. schachtii. In addition, during the course of this experiment, we also measured PR-1 transcript levels in shoots of SAtreated and control plants and found that PR-1 induction was approximately 185-fold higher in shoots of SA-treated plants compared with root tissues (data not shown). This observation may suggest that $A$. thaliana shoot and root tissues differ in their level of responsiveness to exogenously applied SA.

\section{Effects of altered NPR1 function on $\boldsymbol{H}$. schachtii parasitism.}

Given the observed inhibitory effect of SA on $H$. schachtii parasitism, an analysis of mutants involved in SA signaling would help resolve how this inhibition is regulated within the root. The role of NPR1 in regulating SA-dependent defense signaling is well documented (Dong 2004). Mutations in NPRl, also known as NIM1, cause insensitivity to SA and its functional analogues, resulting in an inability of the plant to initiate SAR and upregulate $P R$ gene expression in response to pathogen infection (Cao et al. 1994; Delaney et al. 1995). We investigated the effects of the nprl mutant alleles nprl-1, nprl-2, nprl-3, and nprl-5 on A. thaliana susceptibility to $H$. schachtii. We observed an increased susceptibility phenotype for the nprl-2 and nprl-3 alleles compared with WT (Fig. 2). The nprl-1 and nprl-5 alleles showed $H$. schachtii susceptibilities that were not significantly different from WT (Fig. 2). This disparity in $H$. schachtii susceptibilities between the nprl mutant alleles most likely can be attributed to the varying strengths of the respective alleles. For example, nprl-3 is considered a complete null mutation (Cao et al. 1997) and showed the highest susceptibility to $H$. schachtii of all the alleles tested (Fig. 2). These results suggest that SA-mediated signaling effective against $H$. schachtii is regulated, at least in part, by $N P R I$.

\section{Effects of npr1-suppressor mutations on $\mathrm{H}$. schachtii parasitism.}

The snil and sncl mutants were identified as part of a screen for inducible and constitutive suppressors of $n p r l-1$ (Li
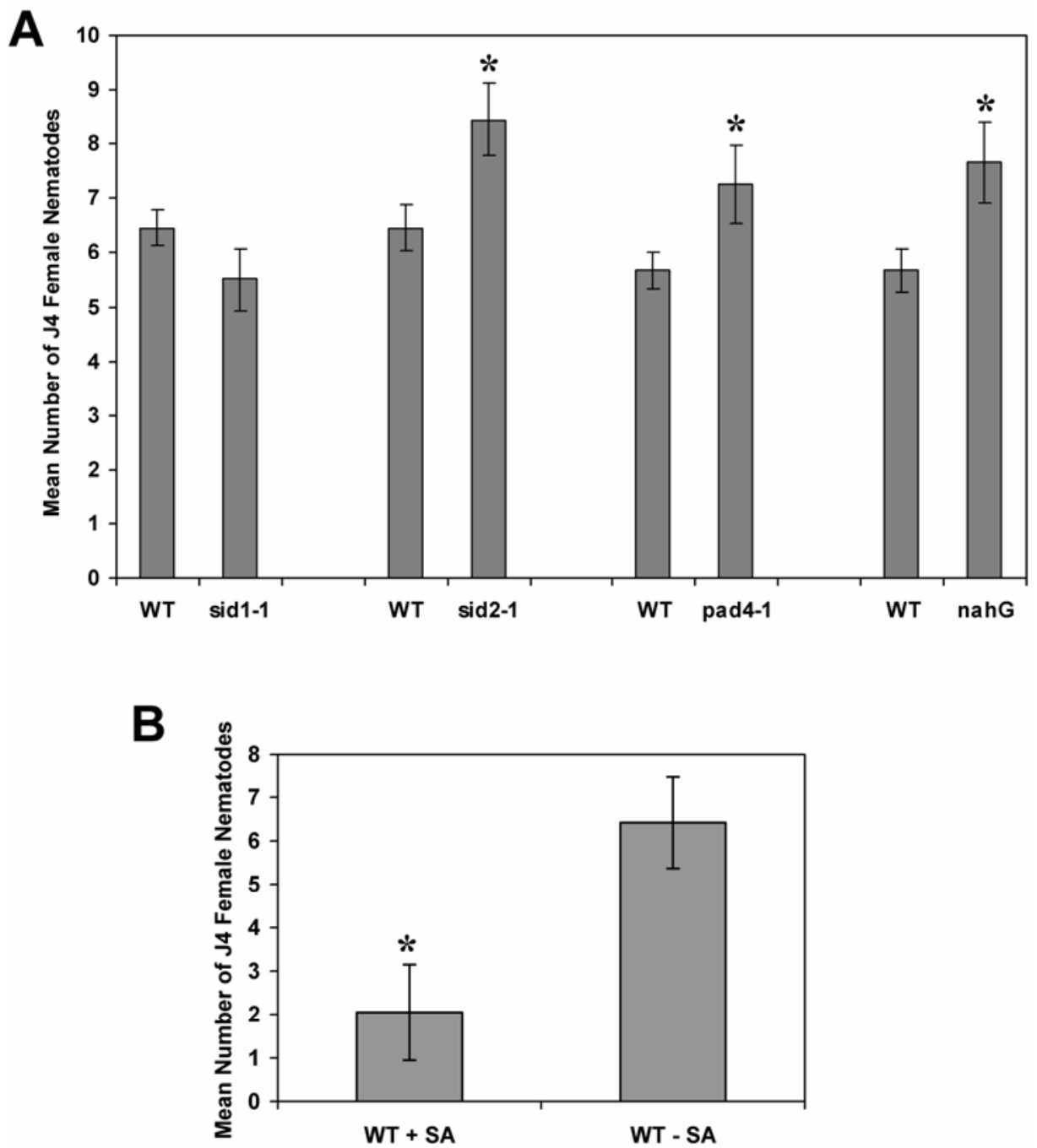

Fig. 1. A, Susceptibilities of salicylic acid (SA)-deficient Arabidopsis mutants to Heterodera schachtii. Shown is the least-squared mean \pm standard error number of fourth-stage juvenile (J4) nematodes for sid1-1 $(n=51)$, sid2-1 $(n=55)$, pad4- $1(n=58)$, and NahG $(n=51)$ compared with wild-type Columbia0 (WT) ( $n=90$ to 103$)$ as determined at 14 days after inoculation. An asterisk $(*)$ indicates that the mutant mean is significantly different $(P<0.05)$ from the corresponding WT mean. B, Effect of SA treatment on Arabidopsis susceptibility to $H$. schachtii. WT plants were germinated and grown on nutrient medium containing either $50 \mu \mathrm{M} \mathrm{SA}(n=17)$ or control solution $(n=19)$. Presented are the least-squared means \pm standard errors from one of three independent experiments. An asterisk $(*)$ indicates that the treatment mean is significantly different $(P<0.05)$ from the control mean. 
et al. 1999 and 2001, respectively). The snilnprl plants regain the ability to respond to exogenous SA or 2-6-dichloro isonicotinic acid (INA), including the accumulation of PR-1 transcript and the induction of SAR following pathogen infection (Li et al. 1999). In contrast, the sncl mutation results in the constitutive activation of a toll interleukin 1 receptor-nucleotide-binding leucine-rich repeat resistance-type gene that appears to modulate both SA-dependent and SA-independent signaling pathways (Zhang et al. 2003). The susceptibilities of sncl and snil plants to $H$. schachtii were determined and compared with WT (Fig. 3). Although sncl plants exhibited a modest decrease in susceptibility to $H$. schachtii, the snil mutant showed a consistent, significantly decreased susceptibility phenotype to the nematode (Fig. 3). The observation that mutation of SNI1, a negative regulator of SA signaling which acts downstream of NPRl (Li et al. 1999), results in decreased $A$. thaliana susceptibility to $H$. schachtii corroborates our discovery of increased $H$. schachtii susceptibility in the sid2-1, pad4,

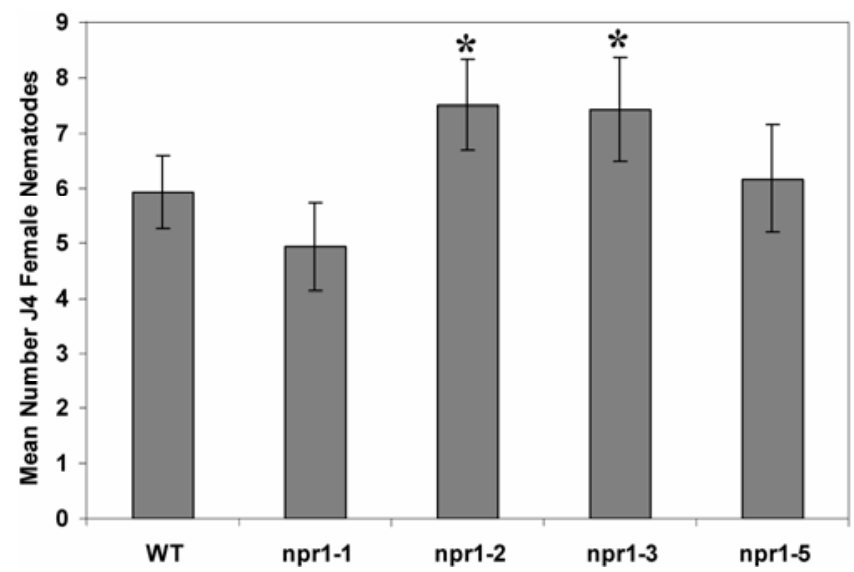

Fig. 2. Susceptibilities of Arabidopsis mutant $n p r l$ alleles to Heterodera schachtii. Shown is the least-squared mean \pm standard error number of fourth-stage juvenile (J4) nematodes for nprl-1 $(n=52)$, nprl-2 $(n=56)$, $n \operatorname{pr} 1-3(n=34)$, and $n \operatorname{pr} 1-5(n=29)$ compared with wild-type Columbia-0 (WT) $(n=73$ to 109$)$ as determined at 14 days after inoculation. An asterisk $(*)$ indicates that the mutant mean is significantly different $(P<0.05)$ from the corresponding WT mean. and nprl mutants, which are nonfunctional positive regulators of SA-mediated signal transduction.

Effects of cpr mutations on $\boldsymbol{H}$. schachtii parasitism.

SA is a strong inducer of PR-1, PR-2, and PR-5 expression (Uknes et al. 1992). Given this fact, and our finding that SA significantly influences $A$. thaliana susceptibility to $H$. schachtii, we ventured to determine whether increased PR gene expression correlates with decreased $H$. schachtii susceptibility. The cprl and cpr6 mutants show constitutive expression of PR-1, PR-2, and PR-5 along with increased levels of endogenous SA in shoot tissues compared with WT (Bowling et al. 1994; Clarke et al. 1998). In addition, cprl and cpr6 previously have been shown to be resistant to virulent isolates of bacterial and fungal foliar pathogens (Bowling et al. 1994; Clarke et al. 1998). Interestingly, neither cprl nor cpr6 exhibited altered susceptibility to $H$. schachtii compared with WT (Fig. 3). This observation suggests that either increased PR gene transcript levels are not directly involved in the SA-mediated inhibition of $H$. schachtii parasitism or the constitutive PR gene expression phenotype of cprl and cpr6 is not manifested in root tissues to the extent that it is in shoots. This latter scenario was addressed experimentally as described below.

\section{Basal PR-1 gene expression is inversely correlated with $H$. schachtii susceptibility.}

Expression of PR-1 in shoot tissues is commonly used as a molecular marker for the activation of SA-mediated responses (Uknes et al. 1992). Because of this trait, we determined the steady-state levels of PR-1 transcript in tissues of sid2-1 (hypersusceptible to $H$. schachtii), cprl (not altered in $H$. schachtii susceptibility), and snil (decreased susceptibility to $H$. schachtii) as an indication of these mutants' inherent level of SA-mediated signaling. Our analysis included a measurement of PR-1 transcript levels specifically in roots for which data regarding SA function and PR gene expression is lacking compared with aboveground plant tissues.

Previous work has demonstrated that PR-1 expression was reduced in sid2-1 shoot organs following pathogen infection relative to infected WT plants (Nawrath and Metraux 1999; Wildermuth et al. 2001). We determined that PR-1 transcript levels in untreated sid2-1 plants were decreased approximately

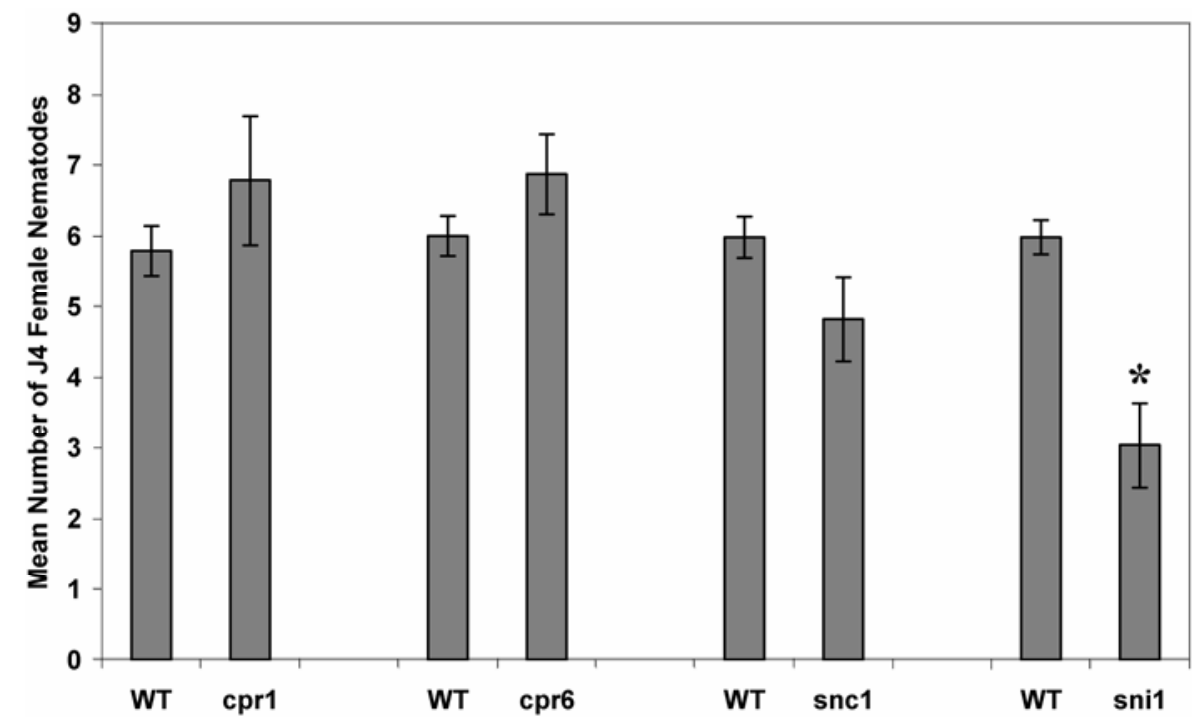

Fig. 3. Susceptibilities of the cprl, cpr6, snil, and sncl Arabidopsis mutants to Heterodera schachtii. Shown is the least-squared mean \pm standard error number of fourth-stage juvenile (J4) nematodes for $\operatorname{cpr} 1(n=31)$, cpr6 $(n=43)$, snc1 $(n=54)$, and snil $(n=39)$ compared with wild-type Columbia-0 (WT) $(n=$ 50 to 104$)$ as determined at 14 days after inoculation. An asterisk $\left(^{*}\right)$ indicates that the mutant mean is significantly different $(P<0.05)$ from the corresponding WT mean. 
180 -fold in shoots and approximately 3 -fold in roots relative to WT tissues (Fig. 4). During the course of our analysis of the sid2-1 mutant, we also determined that, whereas PR-2 and PR5 transcript levels were unaffected in sid2-1 shoots, as previously reported by Nawrath and Metraux (1999), PR-2 transcript levels were decreased approximately 2-fold and PR-5 transcript levels were decreased approximately 6.5-fold in sid2-1 roots relative to WT roots. As was the case with SAtreated WT plants, these results suggest that shoot and root tissues may differ in their regulation of PR gene expression as mediated by SA.

Constitutive PR gene expression in cprl and cpr6 has been observed in shoot tissues; however, it is not known whether this phenotype extends to roots. Given our observation that a loss of SA signaling increases $A$. thaliana susceptibility to $H$. schachtii and that stimulating SA signaling by SA treatment decreases susceptibility, we were surprised that neither cprl nor cpr6 showed altered susceptibility to $H$. schachtii. Potentially explaining this discovery, we found that, although the level of PR-1 transcript was greatly elevated in cprl shoots relative to WT, as has been described previously, it was unaltered in roots of cprl (Fig. 4). In stark contrast, PR-1 transcript levels were significantly elevated in roots of snil, which shows decreased susceptibility to $H$. schachtii versus WT. Taken together, these results indicate that an inverse correlation exists between root PR-1 transcript levels and $H$. schachtii susceptibility.

\section{Temporal expression patterns of PR genes following $\boldsymbol{H}$. schachtii infection.}

In order to obtain a more detailed understanding of the role of SA-mediated defense signaling during $H$. schachtii infection, we used qRT-PCR to measure PR-1, PR-2, and PR-5 transcript levels in WT tissues at three time points following $H$. schachti inoculation. PR-1, PR-2, and PR-5 commonly are used as molecular markers for SA-dependent signaling and consistently have been found to be coordinately regulated by SA (Bowling et al. 1994; Cao et al. 1994; Uknes et al. 1993). WT shoot and root tissues were harvested from $H$. schachtii-infected and control plants 3, 8, and 13 days after inoculation (dai).

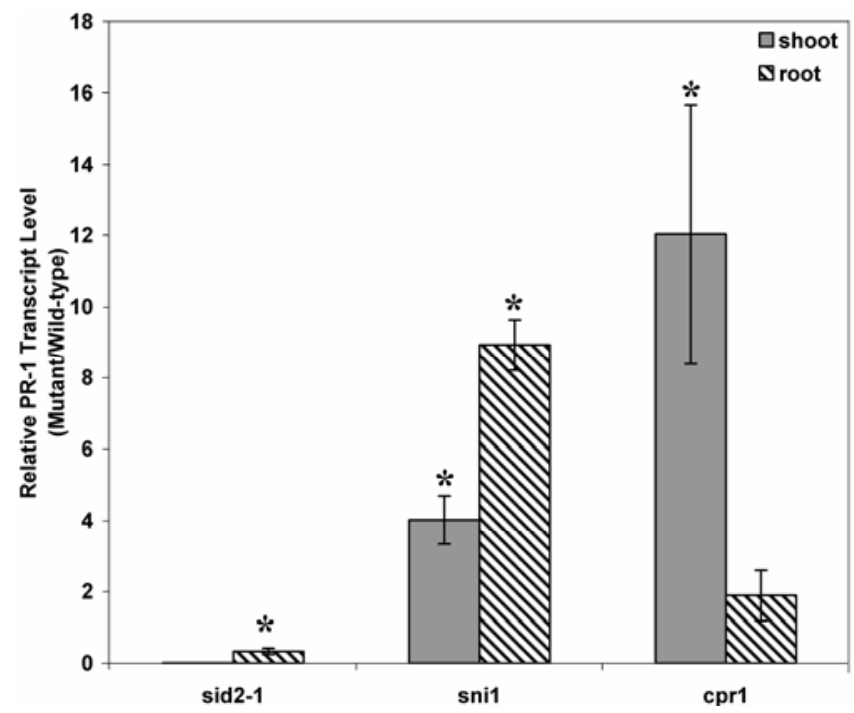

Fig. 4. Pathogenesis-related (PR) 1 transcript levels in shoot and root tissues of sid2-1, snil, and cprl plants relative to wild-type Columbia-0 as measured by quantitative real-time reverse-transcriptase polymerase chain reaction. Presented are mean fold-change \pm standard error in PR1 transcript levels relative to the wild-type Col-0 control tissue (set $=1.0)$ from three biological replicates. An asterisk (*) indicates that the mean fold-change is significantly different from 1.0 as determined by $z$ test $(P<0.05)$.
In our assays, PR-1 transcript levels increased dramatically at 3 dai in shoots of $H$. schachtii-infected WT plants versus the uninoculated control (Fig. 5A). PR-1 transcript levels then progressively declined from 8 to 13 dai. These data suggest that $H$. schachtii infection of A. thaliana roots elicits a classic molecular SAR response in shoot tissues.

In contrast to shoot tissues, roots from $H$. schachtii-infected plants showed no elevation in PR-1 transcript levels at any time point following inoculation (Fig. 5B). PR-2 and PR-5, however, were induced in infected roots, showing similar patterns of induction that reached a maximum at 8 dai and decreased thereafter. Therefore, although $H$. schachtii infection elicits the induction of PR-1 gene expression in distal shoot
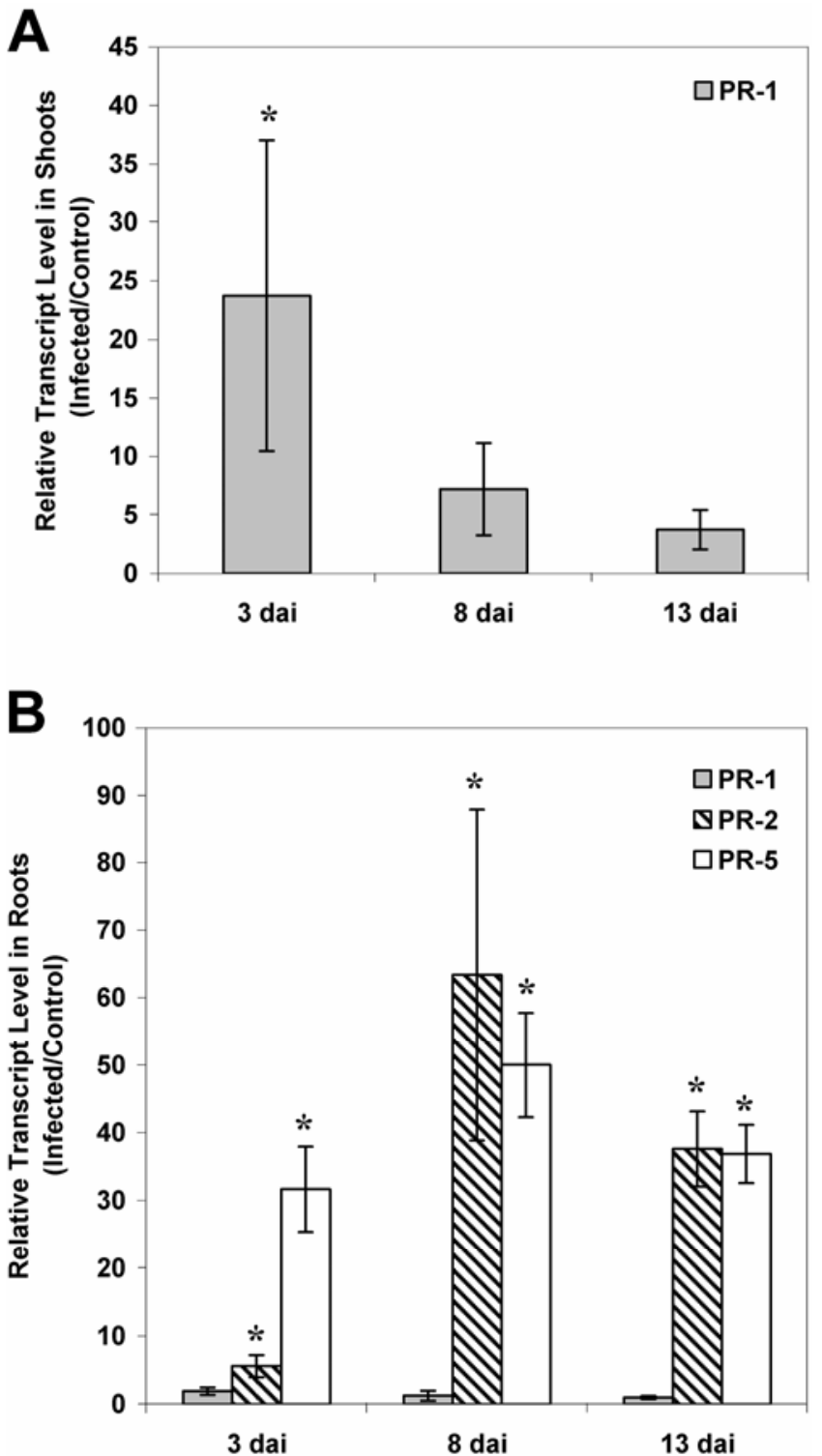

Fig. 5. Time-course analysis of pathogenesis-related (PR) gene expression by quantitative real-time reverse-transcriptase polymerase chain reaction in shoot and root tissues of wild-type Columbia-0 (WT) plants infected with Heterodera schachtii. A, PR1 transcript levels in shoot tissues of WT plants at 3,8 , and 13 days after inoculation (dai) with $H$. schachtii. Presented are mean fold-change \pm standard error in PR1 transcript levels relative to control uninfected tissue (set $=1.0$ ) from four biological replicates. B, PR1, PR2, and PR5 transcript levels in root tissues of WT plants at 3,8 and 13 dai with H. schachtii. Presented are mean fold-change \pm standard error in PR transcript levels relative to control uninfected tissue $(\mathrm{set}=1.0)$ from five to six biological replicates. An asterisk $\left(^{*}\right)$ indicates that the mean fold-change is significantly different from 1.0 as determined by $z$ test $(P<0.05)$. 
tissues, this induction is absent from the infected root tissues. To our knowledge, an uncoupling of PR-1 induction from that of PR-2/PR-5 has been observed only in the SA-deficient sid2 mutant following inoculation with Pseudomonas syringae (Nawrath and Métraux 1999). In that instance, the authors postulated that $P$. syringae infection elicited an SA-independent induction of PR-2 and PR-5 (Nawrath and Métraux 1999). We investigated this scenario in our own system by measuring the fold change in PR-2 and PR-5 transcript levels in WT and sid2-1 roots infected with $H$. schachtii relative to their uninoculated controls. PR-2 and PR-5 induction occurred in sid2

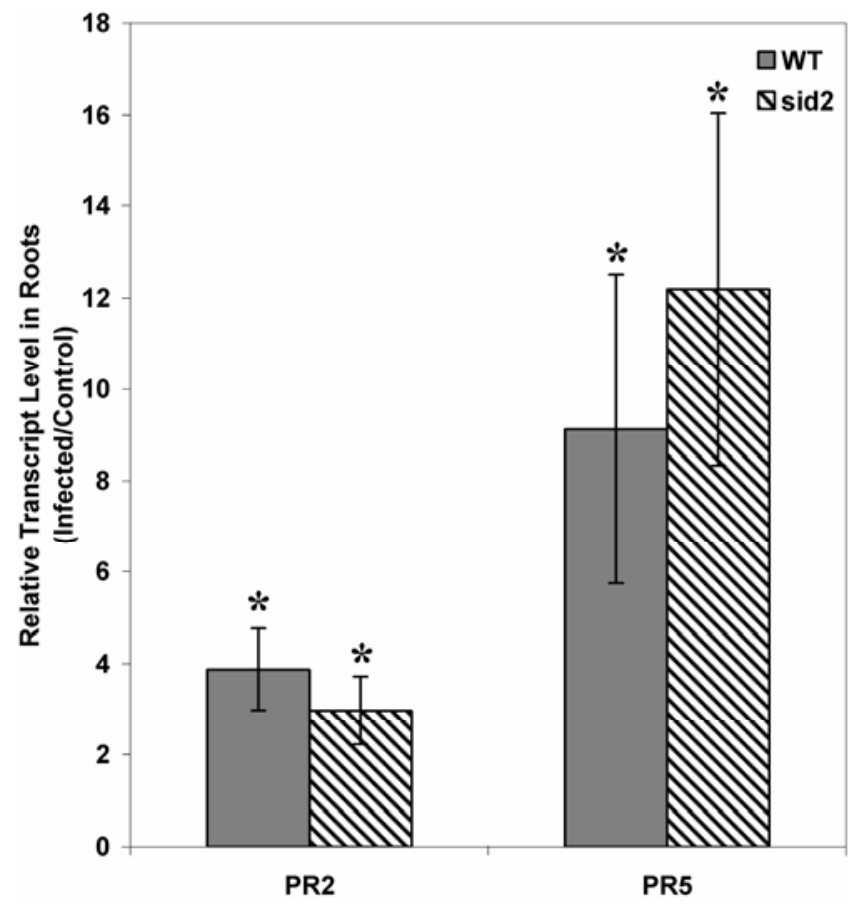

Fig. 6. Induction of pathogenesis-related (PR)-2 and PR-5 transcript levels in sid2-1 and wild-type Columbia-0 (WT) roots following Heterodera schachtii infection. Presented are mean fold-change \pm standard error at 8 days after inoculation with $H$. schachtii relative to control uninfected tissue (set $=1.0$ ) from five biological replicates. An asterisk (*) indicates that the mean foldchange is significantly different from 1.0 as determined by $z$ test $(P<0.05)$. roots following $H$. schachtii infection to approximately the same extent as that observed in WT roots infected with the nematode (Fig. 6). This observation indicates that $H$. schachtii infection elicits an SA-independent induction of PR-2 and PR-5 in roots.

\section{Quantification of total SA}

in wild-type shoots and roots after $\boldsymbol{H}$. schachtii infection.

Our finding that PR-1 transcript levels were elevated in shoots but not roots of $H$. schachtii-infected plants prompted us to quantify endogenous total SA levels in these tissues following infection. Shoot and root tissues of WT plants were harvested from $H$. schachtii-infected and control plants at 4 dai. Total SA was extracted and quantified by HPLC. Results show that total SA levels were slightly elevated in the shoots of $H$. schachtii-infected plants compared with control shoots (Fig. 7). However, this difference was not statistically significant. No change in total SA level was observed in the respective root tissues. Successful $H$. schachtii infection during these experiments was confirmed by observing increased levels of PR-1 transcript in shoot tissues of nematode-infected plants compared with control plants (data not shown).

\section{DISCUSSION}

Sedentary endoparasitic nematodes comprise a large group of damaging plant pathogens that infect and parasitize the roots of their hosts. The interaction between these nematodes and their hosts is highly complex and their obligate root-parasitic nature has proven to be a hindrance toward the molecular characterization of these pathosystems, including the targeted exploration of plant defense responses during nematode parasitism. As a consequence, compared with foliar bacterial, viral, and fungal pathogens, there is a considerable lack of knowledge regarding which defense signaling genes or pathways are effective against plant-parasitic nematodes during a compatible interaction. The research described here aims to provide a broad characterization of the role of SA-mediated defense signaling in the A. thaliana response to infection by the cyst nematode $H$. schachtii. In addition, studies involving plantnematode interactions provide an opportunity to observe defense signaling in root tissues, which is an underrepresented perspective in plant defense research.

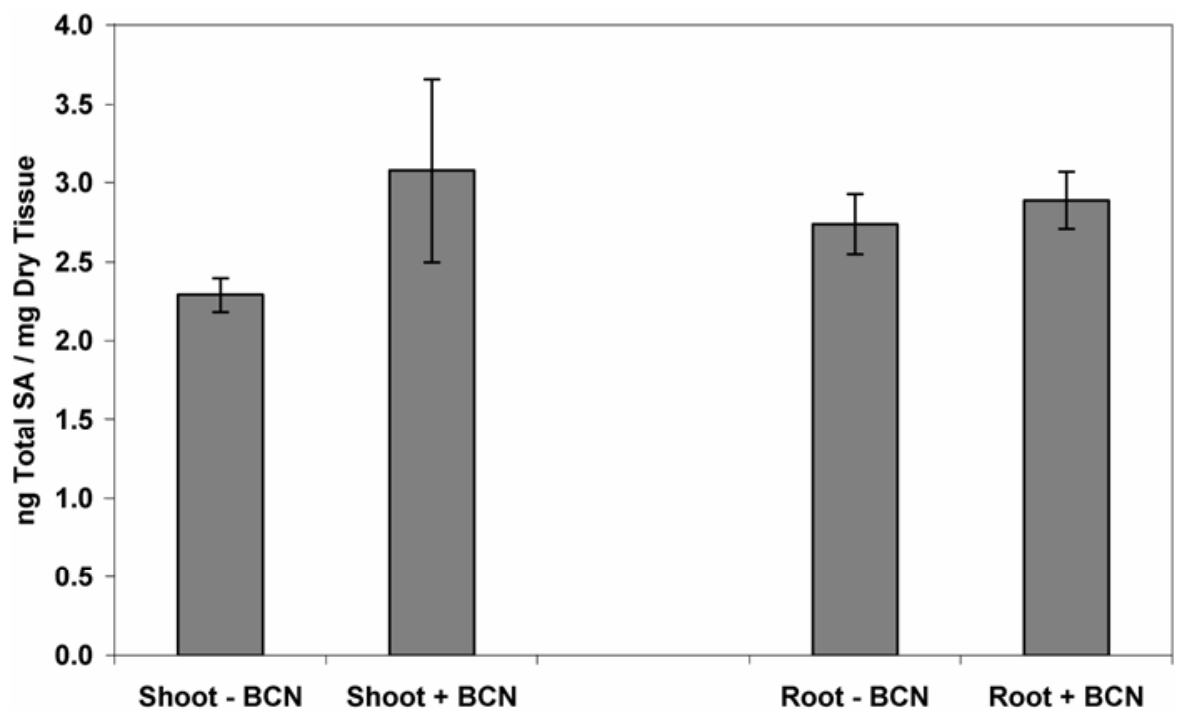

Fig. 7. Quantification of total salicylic acid levels in shoot and root tissues of wild-type Columbia-0 plants 4 days after inoculation with Heterodera schachtii $(\mathrm{BCN})$. Presented are the means \pm standard error of five biological replicates from one representative experiment. No significant difference in total SA levels between $\mathrm{BCN}$-infected and control tissues was detected by paired $t$ test $(P<0.05)$. 
Our analyses of $A$. thaliana mutants perturbed in various aspects of SA-mediated signal transduction revealed that SA is an inhibitor of cyst nematode parasitism during a compatible interaction. Mutants unable to synthesize or accumulate SA (sid2-1, pad4-1, and NahG) showed a consistently increased susceptibility phenotype to $H$. schachtii. Furthermore, we determined that pretreatment of wild-type plants with SA significantly decreased their susceptibility to the nematode while simultaneously inducing PR-1 gene expression in both roots and shoots. Taken together, these data strongly suggest that SAmediated signaling plays a significant role in limiting nematode parasitism during a compatible interaction. Earlier work conducted by Maheshwari and Anwar (1990) suggested that application of SA to tomato plants prior to inoculation with root-knot nematode alleviated root galling; however, this effect of SA was believed to be nematicidal in nature due to the high concentrations of SA that were used in the experiment. For our own studies involving SA treatments, we determined that incubation of $H$. schachtii $\mathrm{J} 2$ in solutions having SA concentrations equivalent to that used for plant treatment had no effect on the nematode's ability to infect and parasitize wild-type plants. Therefore, we believe the observed decreased susceptibility of SA-treated wild-type plants is due to an induction of the SA signaling pathway rather than any nematicidal activity inherent to SA.

NPRl is a key component in plant defense signaling and is required for the induction of SAR elicited either chemically or via pathogen infection. An enormous amount of work has been done toward elucidating the exact mechanism by which NPR1 senses SA accumulation within the cell and transduces this information into defense gene induction (Dong 2004). However, the importance of NPR1 in mediating local defense responses can vary and appears to be dependent on the particular pathogen being studied. For example, nprl-1 plants showed increased susceptibility to the fungal powdery mildew pathogen Erysiphe orontii (Reuber et al. 1998) but not P. syringae maculicola ES4326 (Cao et al. 1994). In our experiments, we found that nprl-2 and nprl-3 but not nprl-1 or nprl-5 showed increased susceptibility to $H$. schachtii. The greatest increase in susceptibility was shown by $n p r l-3$, a nonsense allele of nprl. These data suggest that SA-mediated signaling detrimental to $H$. schachtii is, at least in part, regulated by NPR1.

A role for SA and NPR1 in A. thaliana defense against $H$. schachtii is supported further by our finding that the nprl-suppressor, snil, showed decreased susceptibility to $H$. schachtii. The snil mutant was discovered by screening a mutagenized population of nprl-1 plants that harbored a BGL2-GUS reporter gene. Candidate mutants were identified based on their ability to respond to exogenous elicitors of SAR such as INA (Li et al. 1999). During the characterization of snil, Li and associates (1999) observed that, unlike wild-type plants treated with INA, INA-treated snilNPR1 plants showed intense $\beta$-glucuronidase (GUS) staining in the roots. The author's subsequently showed that snil plants were more sensitive to SA and INA, requiring 10-fold less elicitor than wild type in order to reach maximum $B G L 2-G U S$ reporter gene expression ( $\mathrm{Li}$ et al. 1999). These observations prompted our inquiry into the levels of basal PR-1 transcript in snil roots versus wild-type roots. We found that PR-1 transcript levels were, in fact, greatly elevated in roots of untreated snil plants compared with the untreated wild type. This observation recently has been confirmed by Mosher and associates (2006), who identified a large number of NPR1-dependent genes, including PR-1 and PR-2, which are significantly de-repressed in the snil mutant relative to the wild type. The finding that basal PR-1 expression is higher in snil roots which show decreased susceptibility to $H$. schachtii correlates with our finding that SA-treated wild-type plants, which show PR-1 induction in roots, are less susceptible to the nematode.

As a complement to our analyses of mutants defective in either SA biosynthesis or SA-mediated signaling, we also investigated the effects of constitutive PR gene expression on $H$. schachtii parasitism by measuring the susceptibilities of the cprl and cpr6 mutants. The cprl and cpr6 plants show SA-dependent constitutive expression of PR-1, PR-2, and PR-5 in shoots and are resistant to Peronospora parasitica $\mathrm{NOCO} 2$ and Pseudomonas syringae pv. maculicola ES4326 (Bowling et al. 1994; Clarke et al. 1998). Given our finding that wild-type plants treated with SA prior to $H$. schachtii inoculation showed decreased susceptibility in conjunction with PR-1 gene induction in roots, we were surprised that neither cprl nor cpr6 showed altered susceptibility to the nematode. Reports of constitutive PR gene expression in cprl and cpr6 stem from studies involving shoot tissues or whole seedlings; therefore, it remained unclear to us whether this phenotype extended to root tissues. Upon analysis of basal PR-1 transcript levels in shoots and roots of $c p r l$ and wild-type plants, we determined that, although PR-1 levels were greatly elevated in cprl shoots relative to the wild type as previously reported, there was no such elevation of PR-1 transcript in cprl roots. Coupled with our findings that elevated PR-1 transcript in roots of plants treated with SA or having the snil mutation, these results strongly suggest that a negative correlation exists between PR-1 transcript levels in roots and susceptibility to $H$. schachtii. Although it is unlikely that PR-1 itself has any direct effect on nematode parasitism, PR-1 is one of many genes that are coordinately regulated in plant defense (Maleck et al. 2000) and, therefore, may be viewed as a molecular marker for those genes that are effective against the nematode.

A time-course analysis of PR gene expression in wild-type plants inoculated with $H$. schachtii revealed that PR-2 and PR5 , but not PR-1, are upregulated in roots following nematode infection. However, PR-1 induction was observed in shoots of wild-type plants infected with $H$. schachtii, suggesting that nematode infection elicits a SAR response in the plant, because PR-1 is commonly used as a molecular marker for SAR induction (Bowling et al. 1994; Uknes et al. 1992). This pattern of PR-1 expression between shoot and root tissues agrees with our measurements of total SA levels within shoots and roots collected from $H$. schachtii-infected plants. We observed no difference in total SA levels between infected and uninfected root tissues, with an increase in total SA being present in shoot tissues of infected plants compared with the control. The lack of PR-1 induction and total SA accumulation in $H$. schachtii-infected root tissues suggests that successful cyst nematode parasitism may involve a local suppression of these responses. This hypothesis is supported by recent data concerning $A$. thaliana infection by the root-knot nematode, which also suggests that successful root-knot nematode infection involves a local suppression of the host defense response (Jammes et al. 2005). However, given that our gene expression and total SA quantification analyses were restricted to whole roots, we cannot rule out the possibility that a highly specific induction of PR-1 and accumulation of SA occurs within the immediate area of $H$. schachtii infection.

We were able to determine, via gene expression analyses in $H$. schachtii-infected wild-type and sid2-1 plants, that PR-2 and PR-5 can be induced separately from PR-1 by nematode infection of roots in an SA-independent manner. This phenomenon of uncoupled PR gene expression has been postulated to exist with regards to sid2 infection by $P$. syringae (Nawrath and Métraux 1999). However, to our knowledge, there has yet to be an example of uncoupled PR gene expression in a wild-type plant following pathogen infection as de- 
scribed here regarding the Arabidopsis-cyst nematode compatible interaction.

In addition to results pertinent to plant-nematode interactions, observations made during the course of the above-described experiments suggest that $A$. thaliana shoot and root tissues differ in how they either perceive or respond to SA. In support of this idea, we observed a substantial difference in the level of PR-1 induction following SA treatment in shoots versus roots of wild-type plants even though roots were the primary source of contact during the SA treatment. In addition, our finding that PR-1 transcript levels were unaltered in cprl roots but constitutively expressed at a higher level in shoots further supports this hypothesis. We also routinely observed a significant difference in basal PR-1 transcript levels between shoots and roots of control wild-type plants utilized in our gene expression studies even though the results of our total SA measurement experiments indicate that there is no significant difference in total SA levels between wild-type shoot and root tissues. We believe these observations are compelling and warrant further investigation into this apparent dichotomy between shoots and roots with respect to SA-mediated signal transduction.

In summary, our results strongly indicate a role for SA, mediated by NPR1 and negatively regulated by SNI1, in limiting cyst nematode parasitism during a compatible interaction. The observed decreased susceptibility of the snil mutant to $H$. schachtii, in conjunction with increased root basal PR-1 transcript levels, suggests that defense-related genes effective against the nematode are also more highly expressed in this mutant compared with wild-type plants. A thorough examination of the transcriptional profile of untreated snil plants, as has been recently conducted by Mosher and associates (2006), potentially may identify candidate genes whose direct function is to inhibit parasitism by the nematode.

\section{MATERIALS AND METHODS}

\section{Plant material and growth conditions.}

All $A$. thaliana plants were grown from surface-sterilized seed planted on modified Knops nutrient medium (1.0\% sucrose, $\mathrm{pH}$ 6.4) solidified with $0.8 \%$ Daishin agar (Brunschwig Chemie, Amsterdam, The Netherlands) (Baum et al. 2000) in 9-cm-diameter petri dishes (Fisher Brand; Fisher Scientific, Pittsburg, PA, U.S.A.) or 12-well plates (Falcon Brand; Becton Dickinson, Lincoln Park, NJ, U.S.A.) at $25^{\circ} \mathrm{C}$ under $16 \mathrm{~h}$ of light and $8 \mathrm{~h}$ of darkness. Seed of WT, pad4-1, and sidl-1 were obtained from the Arabidopsis Biological Resource Center at The Ohio State University. Seed of nprl-1, nprl-2, nprl3, nprl-5, snil, snc1, cprl, cpr6, and NahG were a gift from $\mathrm{X}$. Dong at Duke University. Seeds of sid2-1 were a gift from F. Ausubel at Harvard University.

\section{Nematode inoculations and mutant susceptibility data collection.}

Nematode inoculations were performed as previously described (Wubben et al. 2001). Briefly, H. schachtii J2 were hatched from eggs and surface-sterilized by i) washing three times in sterile water, ii) incubating with rotation for $70 \mathrm{~min}$ in $0.001 \%$ chlorhexadiene (Sigma-Aldrich, St. Louis), iii) incubating with rotation for $10 \mathrm{~min}$ in $0.01 \%$ mercuric chloride (Sigma-Aldrich), and iv) washing an additional three times in sterile water. Plants (10 to 12 days old) were inoculated with approximately 300 surface-sterilized J2 nematodes suspended in $37^{\circ} \mathrm{C} 1.5 \%$ low melting point agarose (GIBCO-BRL, Grand Island, NY, U.S.A.). The number of J4 female nematodes that had developed by 14 dai for each plant was determined using a dissecting microscope. The experimental design for assessing mutant susceptibility to $H$. schachtii involved arranging mutant and WT seed within 12-well plates (one seed per well) using a completely randomized design. Each individual mutant was assayed in at least three independent experiments with a minimum replication of 10 plants per experiment. Susceptibility data for each mutant and the corresponding wild-type control were combined across all experiments and analyzed using the PROC MIXED method in SAS (Statistical Analysis Software; SAS Institute, Cary, NC, U.S.A.) to account for unequal replications of mutant and wild-type plants within and between experiments due to experimental design or loss of data because of plant nongrowth or microbial contamination from the nematode inoculation.

\section{SA treatments.}

For the SA treatment experiments, a $1,000 \times$ stock solution of SA (Sigma-Aldrich) was prepared via suspension in $100 \%$ methanol, sterilized by filtration through a $0.22-\mu \mathrm{M}$ filter, and added to autoclaved modified Knops medium to achieve a final SA concentration of $50 \mu \mathrm{M}$. Control medium was prepared in the same manner except that no SA was dissolved in the methanol. Potential adverse effects of SA on $H$. schachtii J2 nematodes were determined via $\mathrm{J} 2$ incubation in three different solutions: i) sterile water, ii) $50 \mu \mathrm{M} \mathrm{SA}$ (prepared using the $1,000 \times$ stock), or iii) control solution containing an equivalent percentage of methanol as the $50 \mu \mathrm{M}$ SA solution. J2 nematodes were incubated overnight at room temperature and then used to inoculate WT plants.

\section{qRT-PCR.}

Total RNA collection, cDNA synthesis, and cycling parameters were conducted as previously described (Wubben et al. 2004).

\section{Total SA quantification.}

Total SA was extracted from freeze-dried shoot and root tissues from WT plants infected or uninfected with $H$. schachtii using a method similar to that described by Dewdney and associates (2000). Briefly, 150 to $300 \mathrm{mg}$ of freeze-dried tissue was suspended in $3 \mathrm{ml}$ of $90 \%$ methanol. The samples were vortexed for $20 \mathrm{~min}$ at room temperature and centrifuged for $20 \mathrm{~min}$ at $1,700 \times g$. The supernatant was transferred to a new tube and the pellet re-extracted with $2 \mathrm{ml}$ of $90 \%$ methanol. The supernatants were combined (approximately $4.5 \mathrm{ml}$ ) and dried down under $\mathrm{N}_{2}$. Then, $500 \mu \mathrm{l}$ of GUS (80 units $/ \mathrm{ml}$ in 0.1 $M$ sodium acetate, $\mathrm{pH}$ 5.2) was added to each sample and vortexed for $5 \mathrm{~min}$. The sample tubes then were covered with aluminum foil and incubated at $37^{\circ} \mathrm{C}$ for $90 \mathrm{~min}$. Following this incubation, $2.5 \mathrm{ml}$ of trichloroacetic acid was added to each sample. Samples were vortexed for $5 \mathrm{~min}$ and then centrifuged for $15 \mathrm{~min}$ at $1,700 \times \mathrm{g}$. The supernatant was transferred to a new tube and extracted two times with $2.5 \mathrm{ml}$ of $1: 1$ ( $\mathrm{vol} / \mathrm{vol})$ ethyl acetate/cyclohexanol. The organic phases were combined and dried down under $\mathrm{N}_{2}$. The samples were stored at $-80^{\circ} \mathrm{C}$ until immediately prior to high-performance liquid chromatography (HPLC) analysis.

For HPLC measurement of total SA, the sample was resuspended in $200 \mu \mathrm{l}$ of methanol and filtered through a $0.22 \mu \mathrm{M}$ filtration column. Then, $20 \mu \mathrm{l}$ was injected into a reversephase $\mathrm{C} 18$ column. The elution program was as follows (flow rate $=1 \mathrm{ml} / \mathrm{min}$ ): 0 to $4.5 \mathrm{~min}, 25 \%$ methanol $/ 85 \% 20 \mathrm{mM}$ potassium acetate, $\mathrm{pH} 5.0 ; 4.5$ to $10.5 \mathrm{~min}$, increase to $70 \%$ methanol; 10.5 to $13.9 \mathrm{~min}, 70 \%$ methanol; 13.9 to $14 \mathrm{~min}$, decrease to $25 \%$ methanol; and 14 to $16 \mathrm{~min}, 25 \%$ methanol. For $\mathrm{SA}$, the excitation wavelength was set to $295 \mathrm{~nm}$ and emission wavelength to $405 \mathrm{~nm}$. The calibration curve for SA was $y=$ $7493.22 x-151592.68\left(R^{2}=0.99\right)$ with an average retention time of approximately $4.9 \mathrm{~min}$. 


\section{ACKNOWLEDGMENTS}

This work was supported by United States Department of AgricultureNational Research Initiative Award \#2003-35301-13504 and by the Iowa State University Plant Sciences Institute. We thank T. Maier (Iowa State University) for his help in conducting the nematode inoculations; D. Boykin and R. Hayes (United States Department of Agriculture-Agricultural Research Service) for their assistance in the statistical analysis of the mutant susceptibility data; X. Dong (Duke University) for her gift of the npr1-1, npr1-2, npr1-3, npr1-5, snil, snc1, cpr1, cpr6, and NahG mutant seed; and F. Ausubel (Harvard University) for his gift of the sid2-1 mutant.

\section{LITERATURE CITED}

Baum, T. J., Wubben, M. J. E., II, Hardy, K. A., Su, H., and Rodermel, S. R. 2000. A screen for Arabidopsis thaliana mutants with altered susceptibility to Heterodera schachtii. J. Nematol. 32:166-173.

Bowling, S. A., Guo, A., Cao, H., Gordon, A. S., Klessig, D. F., and Dong, X. 1994. A mutation in Arabidopsis that leads to constitutive expression of systemic acquired resistance. Plant Cell 6:1845-1857.

Bowling, S. A., Clarke, J. D., Liu, Y. D., Klessig, D. F., and Dong, X. 1997. The cpr5 mutant of Arabidopsis expresses both NPRI-dependent and NPR1-independent resistance. Plant Cell 9:1573-1584.

Cao, H., Bowling, S. A., Gordon, A. S., and Dong, X. 1994. Characterization of an Arabidopsis mutant that is nonresponsive to inducers of systemic acquired resistance. Plant Cell 6:1583-1592.

Cao, H., Glazebrook, J., Clarke, J. D., Volko, S., and Dong, X. 1997. The Arabidopsis NPR1 gene that controls systemic acquired resistance encodes a novel protein containing ankyrin repeats. Cell 88:57-63.

Clarke, J. D., Liu, Y., Klessig, D. F., and Dong, X. 1998. Uncoupling PR gene expression from NPR1 and bacterial resistance: Characterization of the dominant Arabidopsis cpr6-1 mutant. Plant Cell 10:557-569.

Delaney, T. P., Uknes, S., Vernooij, B., Friedrich, L., Weymann, K., Negretto, D., Gaffney, T., Gut-Rella, M., Kessmann, H., Ward, E., and Ryals, J. 1994. A central role of salicylic acid in plant resistance. Science 266:1247-1250.

Delaney, T. P., Friedrich, L., and Ryals, J. A. 1995. Arabidopsis signal transduction mutant defective in chemically and biologically induced disease resistance. Proc. Natl. Acad. Sci. U.S.A. 92:6602-6606.

Dewdney, J., Reuber, T. L., Wildermuth, M. C., Devoto, A., Cui, J., Stutius, L. M., Drummond, E. P., and Ausubel, F. M. 2000. Three unique mutants of Arabidopsis identify eds loci required for limiting growth of a biotrophic fungal pathogen. Plant J. 24:205-218.

Dong, X. 2004. NPR1, all things considered. Curr. Opin. Plant Biol. 7:547-552.

Durrant, W. E., and Dong, X. 2004. Systemic acquired resistance. Annu. Rev. Phytopathol. 42:185-209.

Gaffney, T., Friedrich, L., Vernooij, B., Negrotto, D., Nye, G., Uknes, S., Ward, E., Kessmann, H., and Ryals, J. 1993. Requirement of salicylic acid for the induction of systemic acquired resistance. Science 261:754756.

Glazebrook, J., Rogers, E. E., and Ausubel, F. M. 1996. Isolation of Arabidopsis mutants with enhanced disease susceptibility by direct screening. Genetics 143:973-982.

Glazebrook, J., Chen, W., Estes, B., Chang, H. S., Nawrath, C., Métraux, J.-P., Zhu, T., and Katagiri, F. 2003. Topology of the network integrating salicylate and jasmonate signal transduction derived from global expression phenotyping. Plant J. 34:217-228.

Heck, S., Grau, T., Buchala, A., Métraux, J.-P., and Nawrath, C. 2003. Genetic evidence that expression of NahG modifies defence pathways independent of salicylic acid biosynthesis in the Arabidopsis-Pseudomonas syringae pv. tomato interaction. Plant J. 36:342-352.

Ithal, N., Recknor, J., Nettleton, D., Hearne, L., Maier, T., Baum, T. J., and Mitchum, M. G. 2007. Parallel genome-wide expression profiling of host and pathogen during soybean cyst nematode infection of soybean. Mol. Plant-Microbe Interact. 20:293-305.

Jammes, F., Lecomte, P., de Almeida-Engler, J., Bitton, F., MartinMagniette, M.-L., Renou, J. P., Abad, P., and Favery, B. 2005. Genomewide expression profiling of the host response to root-knot nematode infection in Arabidopsis. Plant J. 44:447-458.

Jirage, D., Tootle, T. L., Reuber, T. L., Frost, L. N., Feys, B. J., Parker, J. E., Ausubel, F. M., and Glazebrook, J. 1999. Arabidopsis thaliana PAD4 encodes a lipase-like gene that is important for salicylic acid signaling. Proc. Natl. Acad. Sci. U.S.A. 96:13583-13588.

Kempster, V. N., Davies, K. A., and Scott, E. S. 2001. Chemical and bio- logical induction of resistance to the clover cyst nematode (Heterodera trifolii) in white clover (Trifolium repens). Nematology 3:35-43.

Li, X., Zhang, Y., Clarke, J. D., Li, Y., and Dong, X. 1999. Identification and cloning of a negative regulator of systemic acquired resistance, SNI1, through a screen for suppressors of $n p r 1-1$. Cell 98:329-339.

Li, X., Clarke, J. D., Zhang, Y., and Dong, X. 2001. Activation of an EDS1-mediated $R$-gene pathway in the sncl mutant leads to constitutive, NPR1-independent pathogen resistance. Mol. Plant-Microbe Interact. 14:1131-1139.

Maheshwari, D. K., and Anwar, M. 1990. Nematicidal activity of some phenolics on root knot, growth and yield of Capsicum frutescens $\mathrm{cv}$. California Wonder. J. Phytopathol. 129:159-164.

Maleck, K., Levine, A., Eulgem, T., Morgan, A., Schmid, J., Lawton, K. A., Dangl, J. L., and Dietrich, R. A. 2000. The transcriptome of Arabidopsis thaliana during systemic acquired resistance. Nat. Genet. 26:403-410.

McDowell, J. M., Williams, S. G., Funderburg, N. T., Eulgem, T., and Dangl, J. L. 2005. Genetic analysis of developmentally regulated resistance to downy mildew (Hyaloperonospora parasitica) in Arabidopsis thaliana. Mol. Plant-Microbe Interact. 18:1226-1234.

Mosher, R. A., Durrant, W. E., Wang, D., Song, J., and Dong, X. 2006. A comprehensive structure-function analysis of Arabidopsis SNI1 defines essential regions and transcriptional repressor activity. Plant Cell 18:1750-1765.

Nandi, B., Kundu, K., Banerjee, N., and Sinha Babu, S. P. 2003. Salicylic acid-induced suppression of Meloidogyne incognita infestation of okra and cowpea. Nematology 5:747-752.

Nawrath, C., and Métraux, J.-P. 1999. Salicylic acid induction-deficient mutants of Arabidopsis express PR-2 and PR-5 and accumulate high levels of camalexin after pathogen inoculation. Plant Cell 11:13931404

Nawrath, C., Heck, S., Parinthawong, N., and Métraux, J.-P. 2002. EDS5, and essential component of salicylic acid-dependent signaling for disease resistance in Arabidopsis, is a member of the MATE transporter family. Plant Cell 14:275-286.

Puthoff, D. P., Nettleton, D., Rodermel, S. R., and Baum, T. J. 2003. Arabidopsis gene expression changes during cyst nematode parasitism revealed by statistical analyses of microarray expression profiles. Plant J. 33:911-921.

Reuber, T. L., Plotnikova, J. M., Dewdney, J., Rogers, E. E., Wood, W., and Ausubel, F. M. 1998. Correlation of defense gene induction defects with powdery mildew susceptibility in Arabidopsis enhanced disease susceptibility mutants. Plant J. 16:473-485.

Rogers, E. E., and Ausubel, F. M. 1997. Arabidopsis enhanced disease susceptibility mutants exhibit enhanced susceptibility to several bacterial pathogens and alterations in PR-1 gene expression. Plant Cell 9:305316.

Sasser, J. N., and Freckman, D. W. 1987. A world perspective on nematology: The role of society. Pages 7-14 in: Vistas on Nematology. J. A Veech and D. W. Dickson, eds. Society of Nematologists, Marceline, MO, U.S.A.

Shah, J., Tsui, F., and Klessig, D. F. 1997. Characterization of a salicylic acid-insensitive mutant (sail) of Arabidopsis thaliana identified in a selective screen utilizing the SA-inducible expression of the tms 2 gene. Mol. Plant-Microbe Interact. 10:69-78.

Uknes, S., Mauch-Mani, B., Moyer, M., Potter, S., Williams, S., Dincher, S., Chandler, D., Slusarenko, A., Ward, E., and Ryals, J. 1992. Acquired resistance in Arabidopsis. Plant Cell 4:645-656.

Wildermuth, M. C., Dewdney, J., Wu, G., and Ausubel, F. M. 2001. Isochorismate synthase is required to synthesize salicylic acid for plant defence. Nature 414:562-571.

Williamson, V. A., and Gleason, C. A. 2003. Plant nematode interactions. Curr. Opin. Plant Biol. 6:327-333.

Williamson, V. A., and Kumar, A. 2006. Nematode resistance in plants: The battle underground. Trends Genet. 22:396-403.

Wubben, M. J. E., II, Su, H., Rodermel, S. R., and Baum, T. J. 2001. Susceptibility to the sugar beet cyst nematode is modulated by ethylene signal transduction in Arabidopsis thaliana. Mol. Plant-Microbe Interact. 14:1206-1212.

Wubben, M. J. E., II, Rodermel, S. R., and Baum, T. J. 2004. Mutation of a UDP-glucose-4-epimerase alters nematode susceptibility and ethylene responses in Arabidopsis roots. Plant J. 40:712-724.

Zhang, Y., Goritschnig, S., Dong, X., and Li, X. 2003. A gain-of-function mutation in a plant disease resistance gene leads to constitutive activation of downstream signal transduction pathways in suppressor of nprl1, constitutive 1. Plant Cell 15:2636-2646. 